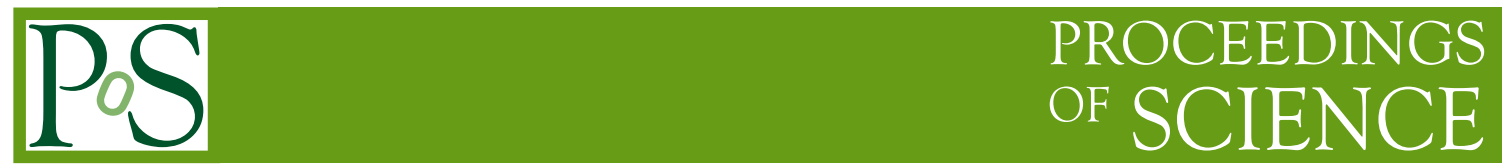

\title{
Search for two-body resonances (theory)
}

\section{Dario Buttazzo*}

Physik-Institut, Universität Zürich

Winterthurerstrasse 190, CH-8057 Zürich, Switzerland

E-mail: buttazzo@physik.uzh.ch

Searches for two-body resonances are a simple and important way to look for new physics at the LHC. In this talk I shall discuss a few examples involving scalar and vector particles, both from a general perspective, and in the context of specific motivated models of new physics at the $\mathrm{TeV}$ scale.

VII Workshop Italiano sulla fisica pp a LHC

16-18 May 2016

Pisa, Italy

${ }^{*}$ Speaker. 
Is the Standard Model (SM) of elementary particles a complete description of physics up to some high energy scale, or are there new states around the Fermi scale? This question is of fundamental importance for particle physics, and drives the numerous searches for new physics at the LHC experiments. A particularly simple and effective class of searches is the one for resonances decaying into two known particles.

The description of these two-body processes is quite simple, since all the physics can be described by the partial decay widths of the resonance, which determine both the decay and the production of the new state $\phi$. For proton collisions at the LHC the production cross-section of a spin- $j$ particle reads

$$
\sigma_{p p \rightarrow \phi}(s)=\frac{2 j+1}{m_{\phi} s} \sum_{\{a b\}} \mathscr{P}_{\{a b\}} \Gamma_{\phi \rightarrow a b},
$$

where $\sqrt{s}$ is the center-of-mass energy, $m_{\phi}$ is the mass of the resonance, and $\mathscr{P}_{\{a b\}}$ is the parton luminosity of the $\{a b\}$ partonic initial state. If the two decay products are equal, the resonance can obviously have only integer spin.

From the experimental point of view, searches for two-body resonances consist in looking for peaks in the invariant mass distributions of the decay products, and their interpretation is therefore usually quite a simple task.

This talk is not intended to be a complete review of the resonance searches performed at the LHC. I shall rather present a brief selection of theoretically motivated examples, including in particular scalar and vector resonances.

\section{Singlet-like Higgs bosons}

The simplest example of a new state decaying into pairs of SM particles is realised adding just a real scalar field, singlet under all the known gauge groups, to the SM. Despite its great simplicity, this scenario is of considerable physical relevance, since it can easily arise in many of the most natural extensions of the SM - e.g. the Next-to-Minimal Supersymmetric SM (NMSSM), Twin Higgs, some Composite Higgs models.

In general, such a singlet will mix with the Higgs boson. As a consequence, both physical scalar states are coupled to SM particles, hence they can both be produced at colliders and be observed by means of their visible decays. In the following, after briefly reviewing the main properties of a generic singlet-like scalar, I shall present the constraints on the existence of such a particle that arise from both direct searches and Higgs couplings precision measurements.

Let us call $h$ and $\phi$ the two neutral, CP-even propagating degrees of freedom, with masses $m_{h}=125.1 \mathrm{GeV}$ and $m_{\phi}$. They are related to the Higgs and singlet gauge eigenstates via a mixing angle $\gamma$. In a weakly interacting theory, the couplings of $h$ and $\phi$ are just the ones of a standard Higgs boson with the same mass, rescaled by a universal factor of $c_{\gamma}$ or $s_{\gamma}$, respectively. As a consequence, their signal strengths $\mu_{h, \phi}$ are

$$
\begin{aligned}
\mu_{h} & =\mu_{\mathrm{SM}}\left(m_{h}\right) \times c_{\gamma}^{2}, \\
\mu_{\phi \rightarrow V V, f f} & =\mu_{\mathrm{SM}}\left(m_{\phi}\right) \times s_{\gamma}^{2} \times\left(1-\mathrm{BR}_{\phi \rightarrow h h}\right), \\
\mu_{\phi \rightarrow h h} & =\sigma_{\mathrm{SM}}\left(m_{\phi}\right) \times s_{\gamma}^{2} \times \mathrm{BR}_{\phi \rightarrow h h},
\end{aligned}
$$



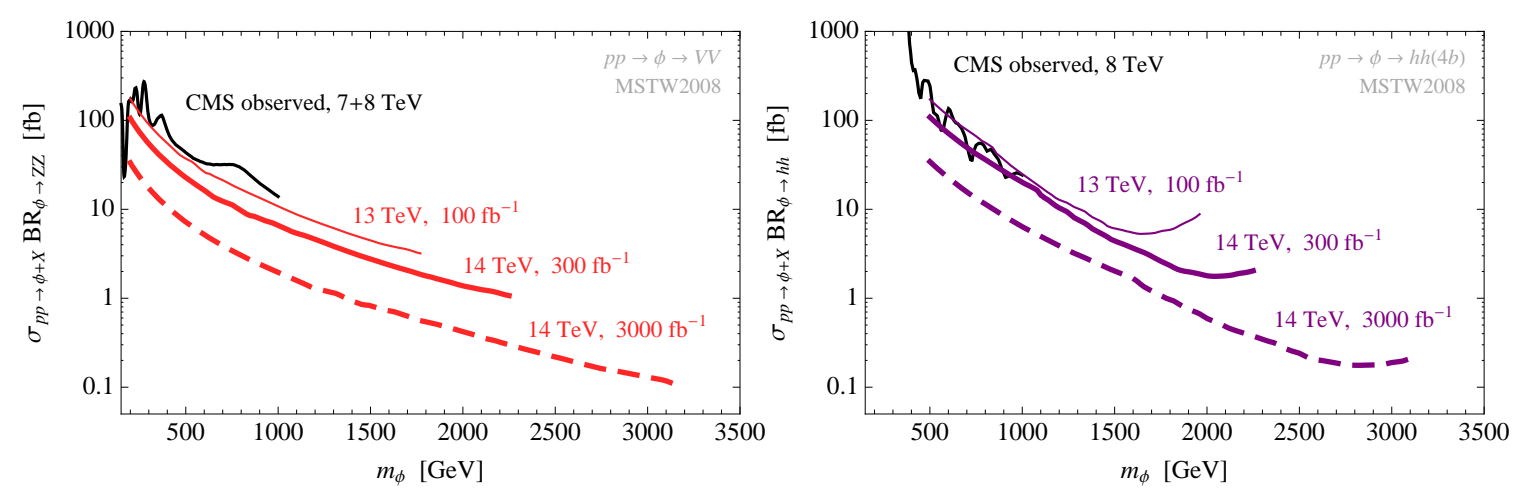

Figure 1: Excluded values and projected reach for $\mu_{\phi \rightarrow Z Z}$ (left) and $\mu_{\phi \rightarrow h h}$ (right). From [1].

where $\mu_{\mathrm{SM}}(m)$ is the corresponding signal strength of a SM Higgs with mass $m$, and $\mathrm{BR}_{\phi \rightarrow h h}$ is the branching ratio of $\phi$ into two $125 \mathrm{GeV}$ Higgs bosons. The phenomenology of the Higgs system is therefore completely described by three parameters: $m_{\phi}, s_{\gamma}$, and $\mathrm{BR}_{\phi \rightarrow h h}$. The second state $\phi$ behaves like a heavy SM Higgs boson, with reduced couplings and an additional decay width into $h h$. Notice that the mixing angle $\gamma$ and $m_{\phi}$ are not independent quantities, since the former has to vanish when the mass tends to infinity. Indeed,

$$
\sin ^{2} \gamma=\frac{M_{h h}^{2}-m_{h}^{2}}{m_{\phi}^{2}-m_{h}^{2}},
$$

where $M_{h h}$ is the first diagonal entry of the mass matrix of the scalar system before diagonalisation, which is proportional to the electroweak scale. The measurement of the Higgs signal strengths provides a constraint on the mixing angle through (1.1). At present, a global fit to $8 \mathrm{TeV}$ LHC data constrain it to be $s_{\gamma}^{2}<0.23$ at $95 \%$ C.L. [2]. More details about Higgs couplings in this context can be found in [1].

The main decay channels of a heavy singlet are into a pair of $W$ and $Z$ vector bosons, or into a pair of Higgs bosons, if kinematically allowed. In the limit of large $m_{\phi}$, the Goldstone boson equivalence theorem sets the relations

$$
\mathrm{BR}_{\phi \rightarrow h h}=\mathrm{BR}_{\phi \rightarrow Z Z}=\frac{1}{2} \mathrm{BR}_{\phi \rightarrow W W} .
$$

The leading corrections to this relation for finite masses depend only on the vacuum expectation value of the singlet, $v_{s}$. Therefore, to a good approximation $m_{\phi}, M_{h h}$, and $v_{s}$ constitute a set of independent parameters that describe the phenomenology. The exact formulae for the $h h h$ and $\phi h h$ couplings as functions of these parameters are reported in [1].

Both the ATLAS and CMS collaborations provide a combined limit from all the $W W$ and $Z Z$ channels, with the strongest bound always coming from searches in the $4 \ell$ and $2 \ell 2 v$ final states [3]. In the di-Higgs channel, the main constraint comes from the $4 b$ final state [4], but $2 b 2 \gamma$ is also important for low masses. All these searches are already sensitive to cross-sections smaller than the ones for a SM Higgs at the same mass, and exceed the reach of Higgs coupling measurements for low enough resonance masses. Projections for the next stages of the LHC and for future colliders have been obtained in [1], rescaling the expected limits from the $8 \mathrm{TeV}$ LHC with 

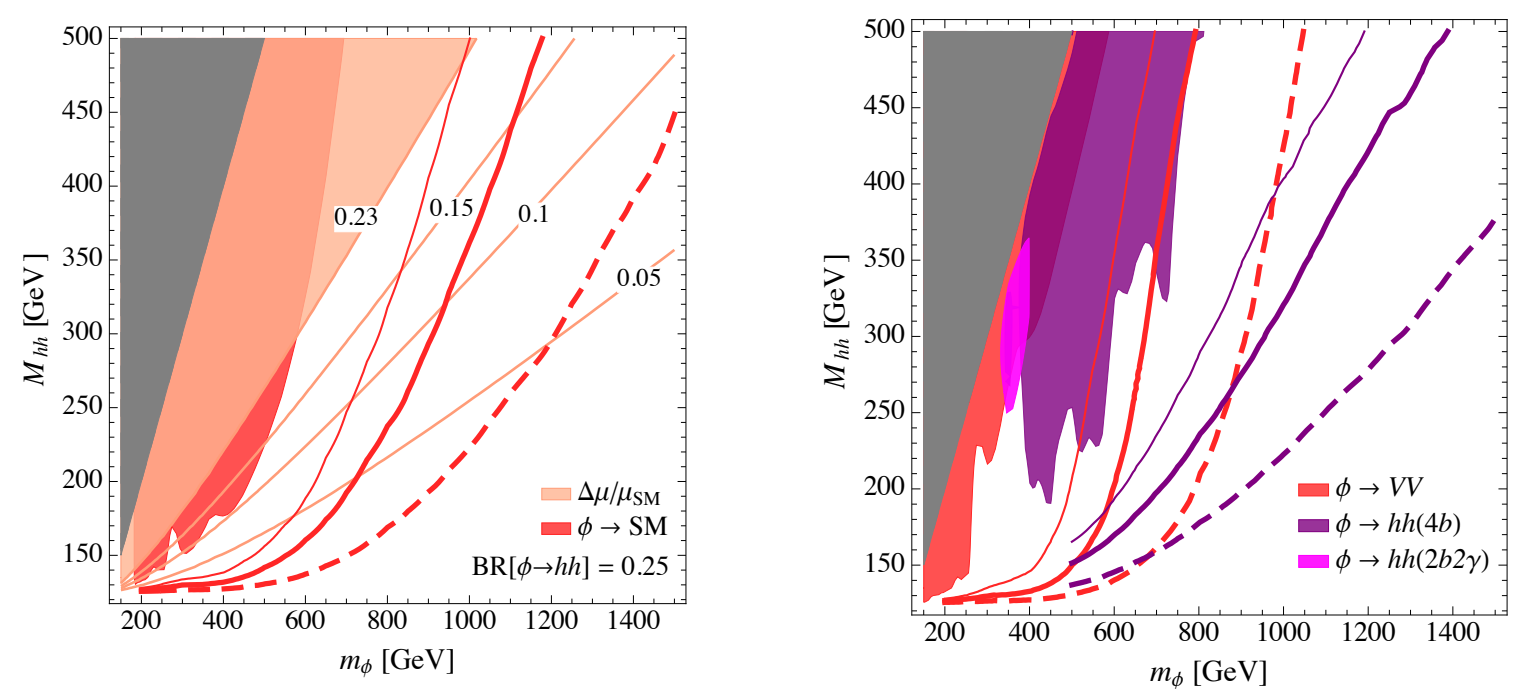

Figure 2: Left: Comparison between the combined reach of direct searches (red) and modifications to Higgs couplings (pink) for a generic singlet in the plane $m_{\phi}-M_{h h} . \mathrm{BR}_{\phi \rightarrow h h}$ has been fixed to 0.25 for simplicity, and the direct exclusion is dominated by $\phi \rightarrow Z Z$. Right: example in which the hh searches (purple and pink) are more sensitive that the ones into $V V(r e d)$. Here $v_{s}=-75 \mathrm{GeV}$. The notation for the direct exclusion lines is the same as in figure 1. Figures from [1].

the parton luminosities of the backgrounds, following the procedure presented in [5]. This method is subject to a number of rather strong assumptions and simplifications, but is nevertheless suited for obtaining a quick estimate of the reach in cross-section with a reasonable level of accuracy. Figure 1 shows the present and extrapolated limits on the $\mu_{\phi \rightarrow V V}$ and $\mu_{\phi \rightarrow h h}$ signal strengths, normalised to SM values of the cross-sections. The sensitivity in the two channels is similar. Figure 2 (left) shows a comparison between direct and indirect searches, but this time in the $m_{\phi}-M_{h h}$ plane, for $\mathrm{BR}_{\phi \rightarrow h h}=1 / 4$. The direct exclusion is dominated by $\phi \rightarrow V V$ due to the larger branching ratio. Figure 2 (right) shows the direct bounds in a particular case where $\mathrm{BR}_{\phi \rightarrow h h}$ is enhanced.

\section{Enhanced production of scalar singlets}

In the previous example all operators of dimension higher than four were neglected. The contributions from these operators, arising from loops, can however significantly affect the predictions for the cross-section and branching ratios of (1.2) if the singlet scalar and the SM fields are coupled to some other degree of freedom.

Let us first consider the gluon-fusion production of a scalar resonance and its decay into gauge bosons (photons, gluons, or electroweak bosons), in the limiting case where all the interactions arise at the loop level, i.e. in the absence of a mixing with the SM Higgs. This is of particular interest in view of the recent hints of an excess of events, reported by both the ATLAS and CMS experiments, in the search for resonances decaying into two photons, around an invariant diphoton mass of about $750 \mathrm{GeV}$ [6]. In the following, the numerical expressions will refer to a resonance mass of $750 \mathrm{GeV}$, but the discussion has a general validity. 
The relevant effective operators for $g g \rightarrow \phi \rightarrow \gamma \gamma$ are

$$
\mathscr{L}^{\mathrm{eff}} \supset c_{G} \frac{\alpha_{s}}{12 \pi m_{\phi}} \phi G_{\mu \nu}^{a} G^{a, \mu v}+c_{W} \frac{\alpha}{4 \pi m_{\phi}} \phi W_{\mu \nu}^{i} W^{i, \mu v}+c_{B} \frac{\alpha}{4 \pi m_{\phi}} \phi B_{\mu v} B^{\mu v},
$$

where $G_{\mu \nu}^{a}, W_{\mu \nu}^{i}$ and $B_{\mu \nu}$ are the colour, weak, and hypercharge field strength tensors, respectively, while $\alpha_{s}$ and $\alpha$ are the strong and electromagnetic coupling constants. The same effective Lagrangian describes also the interactions of a pseudo-scalar with SM particles, upon the replacement $F_{\mu \nu} F^{\mu v} \rightarrow F_{\mu v} F_{\rho \sigma} \varepsilon^{\mu v \rho \sigma}$ for all the field strengths in (2.1). In terms of the coefficients of (2.1), the partial decay widths into gluons and photons are

$$
\begin{aligned}
\Gamma_{\phi \rightarrow \gamma \gamma} & =\frac{m_{S}}{4 \pi}\left(\frac{\alpha c_{\gamma \gamma}}{4 \pi}\right)^{2} \simeq 2.3 \times 10^{-5} c_{\gamma \gamma}^{2} \mathrm{GeV}, \\
\Gamma_{\phi \rightarrow g g} & =\frac{2 m_{S}}{\pi}\left(\frac{\alpha_{s} c_{G}}{12 \pi}\right)^{2} K_{F} \simeq 4.1 \times 10^{-3} c_{G}^{2} \mathrm{GeV},
\end{aligned}
$$

where $c_{\gamma \gamma}=c_{W} \sin ^{2} \theta_{W}+c_{B} \cos ^{2} \theta_{W}, \theta_{W}$ is the Weinberg angle, and $K_{F}$ is a QCD correction factor. The widths into gauge bosons can be related to the one into two photons by one single parameter $R_{W B}=c_{W} / c_{B}[7]$,

$$
\begin{aligned}
\Gamma_{\phi \rightarrow Z \gamma} & =\frac{2\left(1-R_{W B}\right)^{2} \tan ^{2} \theta_{W}}{\left(1+R_{W B} \tan ^{2} \theta_{W}\right)^{2}} \Gamma_{\phi \rightarrow \gamma \gamma}, \\
\Gamma_{\phi \rightarrow Z Z} & =\frac{\left(\tan ^{2} \theta_{W}+R_{W B}\right)^{2}}{\left(1+R_{W B} \tan ^{2} \theta_{W}\right)^{2}} \Gamma_{\phi \rightarrow \gamma \gamma}, \\
\Gamma_{\phi \rightarrow W W} & =\frac{2 R_{W B}^{2}}{\left(\cos ^{2} \theta_{W}+R_{W B} \sin ^{2} \theta_{W}\right)^{2}} \Gamma_{\phi \rightarrow \gamma \gamma} .
\end{aligned}
$$

The production cross-section is also determined from $\Gamma_{\phi \rightarrow g g}$ and, for $m_{\phi}=750 \mathrm{GeV}$ at a center-ofmass energy $\sqrt{s}=13 \mathrm{TeV}$, reads [7] ( $\mathscr{P}_{\text {gg }}$ is the gluon parton luminosity)

$$
\sigma_{p p \rightarrow \phi}^{13 \mathrm{TeV}}=\frac{1}{m_{\phi} S} \mathscr{P}_{g g} \Gamma_{\phi \rightarrow g g} \simeq c_{G}^{2} \times(55 \pm 6) \mathrm{fb} .
$$

A simple realisation of this scenario is obtained coupling a set of heavy coloured and charged fermions $\Psi_{i}$ to the scalar $\phi$. If the new fermions are triplets of colour, with mass $M_{i}$, charge $Q_{i}$, and coupling $g_{i}$ to the scalar, one gets

$$
c_{G}=\sum_{i} g_{i} \frac{m_{\phi}}{M_{i}}, \quad c_{\gamma \gamma}=\frac{2}{3} \sum_{i} g_{i} N_{c} Q_{i}^{2} \frac{m_{\phi}}{M_{i}} .
$$

Figure 3 (left) shows the $95 \%$ C.L. best fit values for $c_{G, B}$ obtained interpreting the ATLAS and CMS $750 \mathrm{GeV}$ excess as a gluon-fusion produced resonance with $\sigma_{p p \rightarrow \phi \rightarrow \gamma \gamma}=4.7_{-1.1}^{+1.2} \mathrm{fb}$ [7]. The blue lines correspond to the predictions from the loops of heavy fermions of different charges. The right panel of the same figure shows instead the predictions for the $Z \gamma, Z Z$, and $W W$ cross-sections at $13 \mathrm{TeV}$ from (2.4) - (2.6) as a function of $R_{W B}$, still fixing the diphoton signal to the same value. If the diphoton signal is not a statistical fluctuation, a generic prediction is that it should show up in at least one of these other bosonic channels - unless $R_{W B}$ is in a very narrow range around one, where all the other branching ratios are small. 

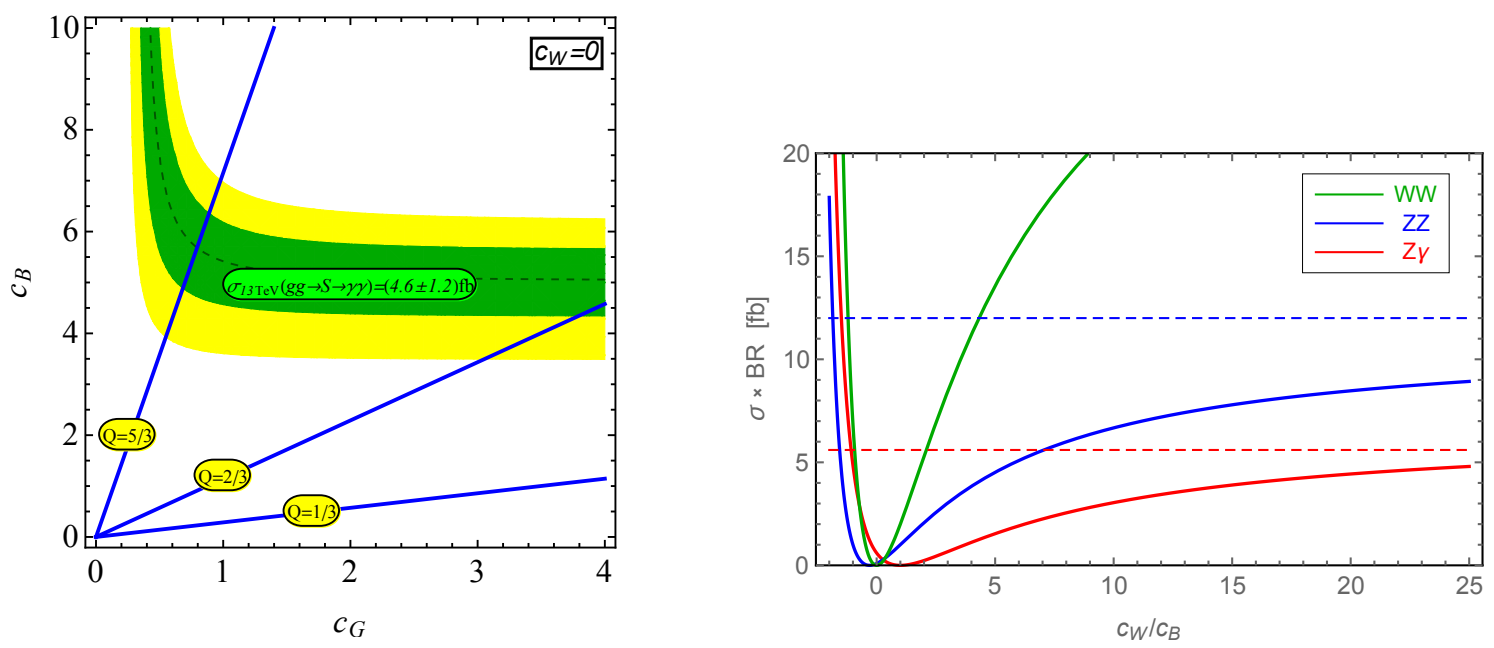

Figure 3: Left: Fit to the diphoton cross-section of a scalar $750 \mathrm{GeV}$ resonance from the ATLAS and CMS excesses, in the plane $c_{G}-c_{B}$. The green and yellow regions are preferred at $68 \%$ and $95 \%$ C.L. The blue lines correspond to the prediction from a heavy quark of charge $Q=1 / 3,2 / 3,5 / 3$ in the loop. Figure from [7]. Right: $Z \gamma, Z Z$, and $W W$ cross-sections (assuming the best fit value for the $\gamma \gamma$ cross-section) as functions of $c_{W} / c_{B}$. The dashed lines are the present experimental exclusions.

The Lagrangian (2.1) can be generalised as follows to include also renormalisable couplings to the SM fields:

$$
\mathscr{L}=c_{V} \frac{\phi}{m_{\phi}}\left(m_{Z}^{2} Z_{\mu} Z^{\mu}+2 m_{W}^{2} W_{\mu}^{+} W^{-\mu}\right)+c_{f} \frac{\phi}{m_{\phi}} m_{f} \bar{f} f+\frac{\phi}{m_{\phi}}\left(c_{\partial h} \partial_{\mu} h \partial^{\mu} h-c_{h} \frac{m_{h}^{2}}{2} h^{2}\right) .
$$

The tree-level decay widths which follow from these couplings are

$$
\begin{aligned}
\Gamma_{\phi \rightarrow Z Z(W W)} & =(2) \frac{c_{V}^{2} m_{\phi}}{32 \pi} \sqrt{1-\beta_{Z(W)}}\left(1-\beta_{Z(W)}+\frac{3}{4} \beta_{Z(W)}^{2}\right) \simeq 6.8(13.9) c_{V}^{2} \mathrm{GeV}, \\
\Gamma_{\phi \rightarrow h h} & =\frac{m_{\phi}}{32 \pi}\left(c_{\partial h}\left(1-\frac{\beta_{h}}{2}\right)+c_{h} \frac{\beta_{h}}{4}\right)^{2} \sqrt{1-\beta_{h}} \simeq 6.3\left(c_{\partial h}+0.029 c_{h}\right)^{2} \mathrm{GeV}, \\
\Gamma_{\phi \rightarrow f \bar{f}} & =\frac{c_{f}^{2} N_{c}}{8 \pi m_{\phi}} \bar{m}_{f}^{2}\left(1-\beta_{f}\right)^{3 / 2}\left(1+\Delta_{\mathrm{QCD}}\right) \stackrel{f=t}{\simeq} 3.3 c_{t}^{2} \mathrm{GeV},
\end{aligned}
$$

where $\beta_{i}=4 m_{i}^{2} / m_{\phi}^{2}$ and $\Delta_{\mathrm{QCD}}$ is a radiative correction factor. The scenario of section 1 of a real singlet mixed with the Higgs boson corresponds to the case where $c_{V}=c_{f}=\sin \gamma m_{\phi} / v$, $c_{\partial h}=0$, while $c_{h}$ can be related to $v_{s}$. For a pseudo-scalar resonance, on the other hand, one has $c_{V}=c_{h}=0$, and only tree-level decays into fermions are allowed. For coefficients $c_{i}$ of order one the branching ratios into vector and Higgs bosons are clearly dominant, and those of (2.2) - (2.4) are strongly suppressed. However, the production cross-section can be significantly enhanced by the contribution of (2.3), and as a consequence the experimental bounds from resonance searches can become more relevant. Using the total width $\Gamma_{\phi}$ as a free parameter, the signal strength of a $750 \mathrm{GeV}$ resonance into diphotons reads

$$
\mu_{\phi \rightarrow \gamma \gamma}^{13 \mathrm{TeV}} \simeq 6.3 \times 10^{-5}\left(\frac{20 \mathrm{GeV}}{\Gamma_{\phi}}\right) c_{G}^{2} c_{\gamma \gamma}^{2} \mathrm{fb},
$$



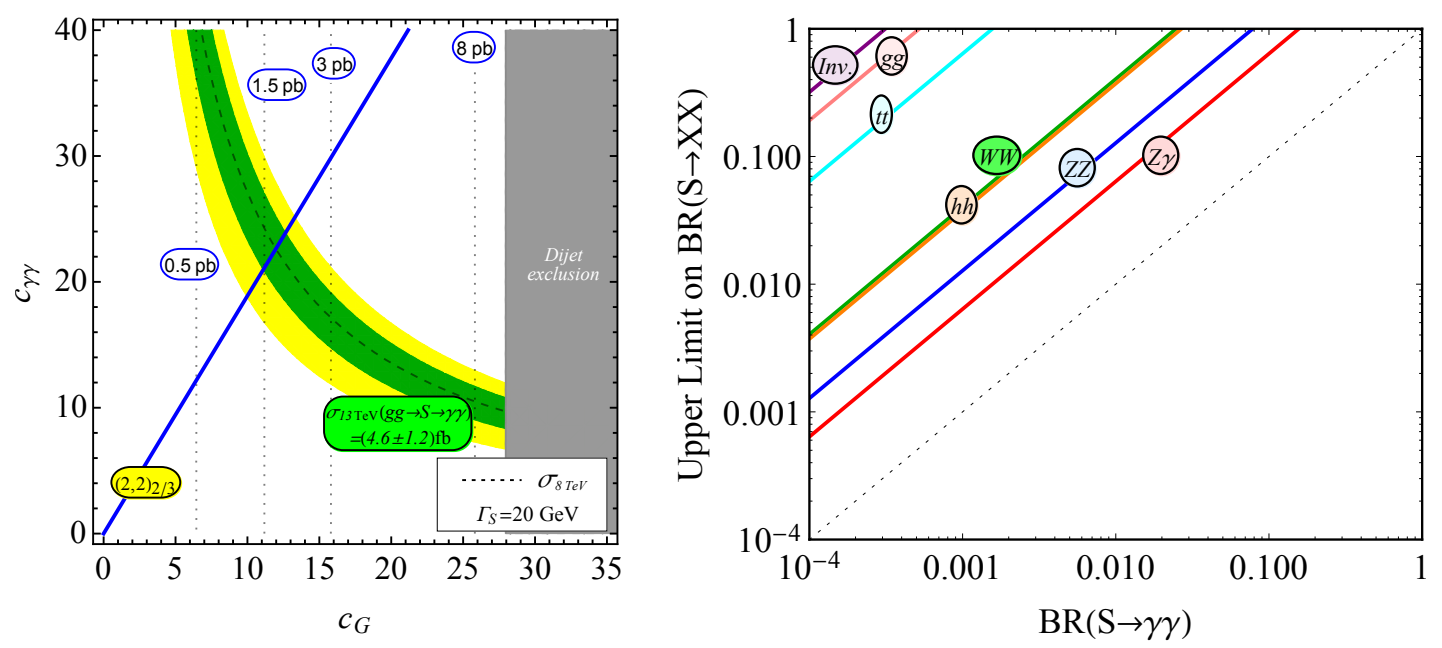

Figure 4: Left: Fit to the diphoton cross-section from the ATLAS and CMS excesses, in the plane $c_{G}-c_{\gamma \gamma}$, for a fixed width of the scalar resonance. The blue line is the prediction for a heavy quark multiplet, bidoublet under $\mathrm{SU}(2)_{L} \times \mathrm{SU}(2)_{R}$, the dotted black lines show the total cross-section. Right: upper limits on different branching ratios of the scalar, from the experimental limits in the various channels, and assuming the best-fit cross-section into diphotons. From [7].

and similarly those into the other tree-level channels are, at $\sqrt{s}=8 \mathrm{TeV}$,

$$
\begin{aligned}
\mu_{\phi \rightarrow Z Z} & \simeq 4.0\left(\frac{20 \mathrm{GeV}}{\Gamma_{\phi}}\right) c_{G}^{2} c_{V}^{2} \mathrm{fb}<12 \mathrm{fb}, \\
\mu_{\phi \rightarrow W W} & \simeq 8.2\left(\frac{20 \mathrm{GeV}}{\Gamma_{\phi}}\right) c_{G}^{2} c_{V}^{2} \mathrm{fb}<38 \mathrm{fb}, \\
\mu_{\phi \rightarrow h h} & \simeq 3.7\left(\frac{20 \mathrm{GeV}}{\Gamma_{\phi}}\right) c_{G}^{2}\left(c_{h \partial}+0.029 c_{h m}\right)^{2} \mathrm{fb}<35 \mathrm{fb}, \\
\mu_{\phi \rightarrow t \bar{t}} & \simeq 2.0\left(\frac{20 \mathrm{GeV}}{\Gamma_{\phi}}\right) c_{G}^{2} c_{t}^{2} \mathrm{fb}<0.6 \mathrm{pb} .
\end{aligned}
$$

The r.h.s. of the previous relations are the experimental bounds from the $8 \mathrm{TeV}$ run of the LHC on each individual channel, which are still stronger than the limits from the $13 \mathrm{TeV}$ run for masses below about a TeV. Figure 4 (left) shows the result of the fit to the ATLAS and CMS excess for $\Gamma_{\phi}=20 \mathrm{GeV}$. The dashed lines indicate the total production cross-section: using the bounds in (2.14) - (2.17) one can immediately read off the limits on the various branching ratios in each point. The grey area is excluded by dijet searches, which depend only on $c_{G}$. Figure 4 (right) shows the upper bounds on the different branching ratios, fixing the diphoton signal strength to its best-fit value. If the width of the hypothetical $750 \mathrm{GeV}$ state is large, the only channels which can account for it are $t \bar{t}$ or invisible decays.

Finally, one can show that couplings to SM particles alone can not account for a large diphoton cross-section: for the case of the top quark, as an example, a coefficient $c_{t} \simeq 50$ would be required, which corresponds to a huge unphysical width into $t \bar{t}$ of several TeV. This means that the observation of a diphoton resonance with sizeable cross-section implies the presence of a whole new sector of charged and coloured particles coupled to it [7]. 

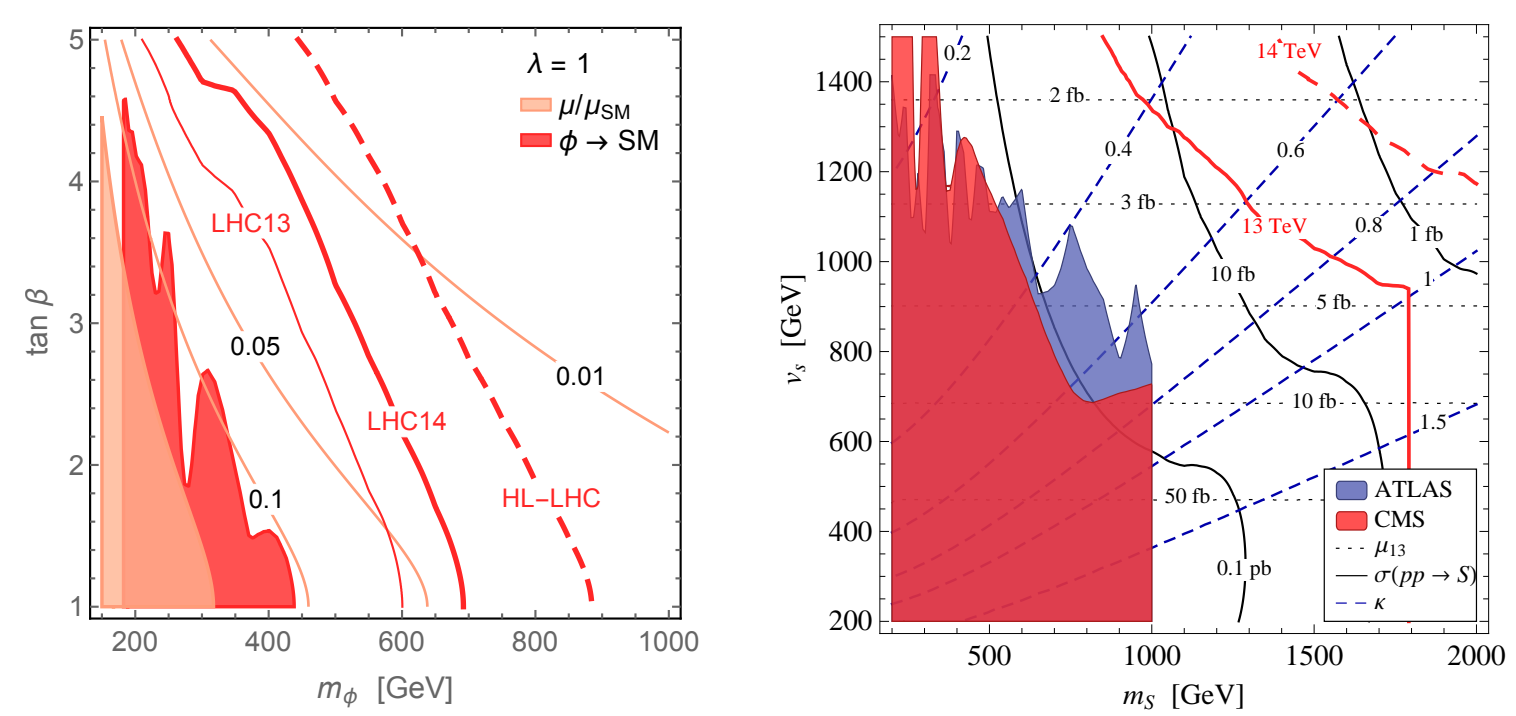

Figure 5: Exclusions and projections for the real scalar of the NMSSM decaying into ZZ in two different NMSSM scenarios. Left: general NMSSM with $\lambda=1$, in the plane $m_{\phi}-\tan \beta$. The LHC exclusion is in red, the Higgs couplings exclusion and projection is in pink. Figure from [1]. Right: NMSSM with vector-like matter in the plane $m_{\phi}-v_{s}$. The coloured regions are the ATLAS (blue) and CMS (red) exclusions. The solid black lines are the $8 \mathrm{TeV} \mathrm{pp} \rightarrow \phi$ cross-section, the blue dashed isolines are for the parameter $\kappa$, the black dotted lines show the diphoton signal of the pseudo-scalar. Figure from [9].

\section{An explicit example: supersymmetry}

A typical example of a class of models which require more than one scalar state is supersymmetry. The Higgs sector of the NMSSM contains the two usual doublets $H_{u, d}$, plus a singlet scalar $S$, coupled through a Yukawa interaction $\lambda H_{u} H_{d} S$ in the superpotential. This coupling $\lambda$ generates an extra tree-level contribution to the Higgs mass is generated, reducing the size of the radiative correction needed to obtain $125 \mathrm{GeV}$. At the same time, the fine-tuning of the electroweak scale $v$ is reduced.

In the decoupling limit for the heavy doublet, the CP-even states are the SM Higgs and the singlet, and can be matched to the general scenario of section 1 via [8]

$$
M_{h h}^{2}=m_{Z}^{2} \cos ^{2} 2 \beta+v^{2} \lambda^{2} \sin ^{2} 2 \beta+\Delta^{2},
$$

where $\Delta$ is the radiative correction and $\tan \beta=v_{u} / v_{d}$. Figure 5 (left) shows the current exclusions and projections from both direct searches and Higgs couplings, in the plane $m_{\phi}-\tan \beta$, for fixed values of $\lambda=1$ and $\Delta=70 \mathrm{GeV}$. It is clear that - even more than in the case of a generic singlet - direct searches for the new resonances are the most powerful probe of the extended Higgs sector of the NMSSM, and always dominate over Higgs coupling measurements.

According to the discussion of section 2, the presence of additional matter will affect the production and decay of the scalar states. One can envisage a simple and predictive scenario with one extra vector-like generation of matter coupled to the singlet $\mathrm{S}$ through a scale invariant Lagrangian

$$
\mathscr{L}=\mathscr{L}_{\mathrm{MSSM}}+\lambda S H_{u} H_{d}+\sum_{i} \lambda_{i} S \bar{\Psi}_{i} \Psi_{i}+\kappa S^{3}
$$


Interestingly, if the singlet becomes strongly coupled at a scale $\Lambda \sim 10^{3 \div 6} \mathrm{GeV}$ (and all its couplings become large at the same scale), the low-energy value of the $\lambda$ coupling is automatically of the right size to predict a $125 \mathrm{GeV}$ Higgs mass, and the masses of the new fermions $M_{i} \sim \lambda_{i} v_{s}$ is around the $\mathrm{TeV}$ [9]. The pseudo-scalar singlet, which will not mix with the Higgs boson if $\mathrm{CP}$ is conserved, can have a large decay rate into photons and can even explain the LHC excess in that channel. The larger cross-section of the real scalar, on the other hand, pushes its mass to higher values to satisfy experimental constraints, as is shown in the right panel of figure 5 .

\section{Vector bosons}

A different class of high-mass two-body resonances that can be searched for at the LHC is the one of vector bosons - e.g. vector triplets such as $W^{\prime}$ or $Z^{\prime}$.

In general the vectors $\rho_{\mu}$ can couple to SM fermions, as well as to the Higgs boson and to the $\mathrm{SM}$ vectors. Let us consider for definiteness a vector triplet of $\mathrm{SU}(2)_{L}$. A simplified Lagrangian can be written as (see e.g. [10])

$$
\mathscr{L}_{\rho}=-\frac{1}{4} D_{[\mu} \rho_{v]}^{a} D^{[\mu} \rho^{v], a}+\frac{m_{\rho}^{2}}{2} \rho_{\mu}^{a} \rho^{\mu, a}+i g_{H} \rho_{\mu}^{a}\left(H^{\dagger} \tau^{a} \stackrel{\leftrightarrow}{D_{\mu}} H\right)+g_{F} \rho_{\mu}^{a} J^{\mu, a}
$$

where $\tau^{a}$ are the Pauli matrices, and $J_{\mu}^{a}$ is a fermionic $\mathrm{SU}(2)_{L}$ current. The coupling $g_{H}$ controls the $\rho$ decays into bosonic channels (vectors and Higgs), while $g_{F}$ is responsible for both the Drell-Yan resonance production and for its fermionic decays. The related decay widths read, in the limit of large $m_{\rho}$,

$$
\begin{aligned}
\Gamma_{\rho^{ \pm} \rightarrow \psi \bar{\psi}} & \simeq 2 \Gamma_{\rho^{0} \rightarrow \psi \bar{\psi}} \simeq N_{c} g_{F}^{2} \frac{m_{\rho}}{96 \pi}, \\
\Gamma_{\rho^{0} \rightarrow W^{+} W^{-}} & \simeq \Gamma_{\rho^{0} \rightarrow Z h} \simeq \Gamma_{\rho^{ \pm} \rightarrow W Z} \simeq \Gamma_{\rho^{ \pm} \rightarrow W^{ \pm} h} \simeq g_{H}^{2} \frac{m_{\rho}}{192 \pi} .
\end{aligned}
$$

Both the couplings to bosons and to light fermions also induce a contribution to the electroweak precision observables, as well as modifications in the coupling of the gauge bosons to fermions, which are strongly constrained by LEP measurements. Furthermore, the exchange of a heavy $\rho$ generates four-fermion interactions, which are again constrained by LEP and by flavour observables. Already from this, the mass of the new vectors is constrained to be in the multi-TeV range, unless the couplings are small. If $c_{H}$ is of $\mathrm{O}(1)$, the main decay channel can be the one into vector bosons: for high masses, boosted $W W$ and $Z Z$ searches are relevant [11], and in this case the $13 \mathrm{TeV}$ results are already stronger than the ones from the $8 \mathrm{TeV}$ run.

An interesting and slightly different scenario is the one where the vectors couple mainly to fermions of the third generation, compatibly with the approximate flavour symmetry exhibited by the quark sector of the SM. Indeed, in this case the four-fermion operators could be related to a number of anomalies in rare decays of $B$ and $K$ mesons, which seem to point to a violation of lepton flavour universality. It has been shown in [12] that a vector triplet with a mass of about $2 \mathrm{TeV}$ and a large coupling to fermions provides a good explanation of the anomalies. If these vectors are composite resonances of a strongly coupled sector, similar to the $\rho$ in QCD, one expects in general pseudo-scalar Goldstone states of lower mass. These can decay to photons in a fashion similar to $\pi^{0} \rightarrow \gamma \gamma$ and even reproduce the LHC diphoton excess [13]. 

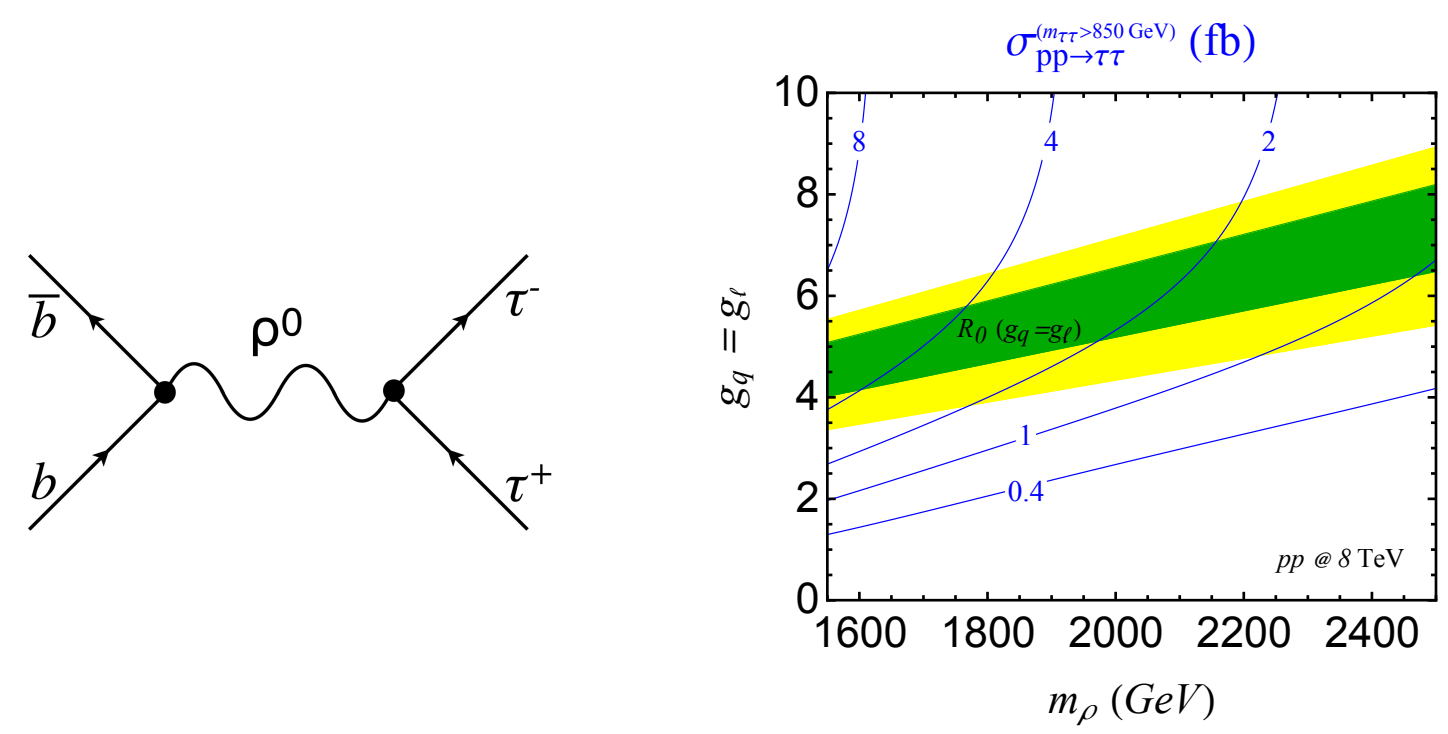

Figure 6: Left: production and decay of a $\rho_{\mu}$ resonance coupled to third generation fermions. A similar diagram also contributes at low energy to the $B_{s} \rightarrow \tau^{+} \tau^{-}$anomaly. Right: cross-section for $p p \rightarrow \rho \rightarrow$ $\tau^{+} \tau^{-}$, with $m_{\tau \tau}>850 \mathrm{GeV}$ corresponding roughly to the experimental signal region. The green and yellow bands are the $68 \%$ and $95 \%$ C.L. regions of the fit to flavour observables. From [13].

The main decay channel of the vectors is into $\tau^{+} \tau^{-}$and $b \bar{b}$, with the former channel being the most sensitive at the LHC. Because of the large coupling required by the flavour constraints, the resonances are expected to be very broad (with a width-to-mass ratio that can be as large as $50 \%$ ). The main production mechanism is through $b \bar{b}$ fusion, despite the small corresponding parton luminosity, due to the large coupling to third generation fermions. For the masses that fit best the flavour and diphoton anomalies, the results from the $8 \mathrm{TeV}$ run still allow this scenario, but are expected to probe it very soon, as the data collected in the new run increase. Figure 6 (right) shows the predicted cross-section for $p p \rightarrow \rho \rightarrow \tau^{+} \tau^{-}$with a $\tau^{+} \tau^{-}$invariant mass larger than $850 \mathrm{GeV}$ (the signal region in the ATLAS search [14] corresponds to a transverse mass larger than that value). The present exclusion for a narrow resonance of high mass is $4 \mathrm{fb}$, and increases to $7 \mathrm{fb}$ for the case of a moderate width. If the coupling to quarks for some reason is larger than the coupling to leptons, $b \bar{b}$ searches can also become relevant. Note that CMS shows a moderate excess of events around a mass of $1.9 \mathrm{TeV}$ in the search for resonances decaying into (b-enriched) dijets [15].

To conclude, it is worth to stress that a signal in high-mass $\tau^{+} \tau^{-}$(or $b \bar{b}$ ) searches is a generic prediction if some new state is involved in the solution of the flavour anomalies, even beyond the model considered here.

\section{Conclusions}

The searches for new resonances at the LHC are a very powerful tool to explore the physics in the $\mathrm{TeV}$ range.

Scalar (and pseudo-scalar) particles appear in most natural extensions of the SM. A general description that goes beyond the validity of a specific model can be performed in the simplest 
cases, such as the one of a scalar singlet. Here two different scenarios were discussed: in the first one the phenomenology of the singlet is purely determined by its mixing with the Higgs boson, while in the second one radiative contributions from additional particles play a dominant rôle in enhancing cross-section and branching ratios. This last case is of interest in view of the possible hits of some new resonance decaying into photons that was recently suggested by the ATLAS and CMS experiments. Bosonic decay channels are the most interesting ones to look at in all the cases discussed.

Vector bosons are usually expected at somewhat higher masses, due to the constraints from LEP precision measurements. Decays into SM vectors are also here important if the new vector bosons couple to the Higgs field. On the other hand, it is interesting to consider different possibilities: a particular phenomenology is obtained if the vectors couple dominantly to fermions of the third family. This possibility is intriguing because it could be related to a number of anomalies in flavour physics.

In many of the channels discussed here, the strongest bounds still come from the first run of the LHC at $8 \mathrm{TeV}$, especially for lower resonance masses. The LHC is however collecting new data at an impressive rate, and it is already starting to probe an unexplored territory.

Acknowledgements: This work was supported in part by the Swiss National Science Foundation (SNF) under contract 200021-159720.

\section{References}

[1] D. Buttazzo, F. Sala and A. Tesi, JHEP 1511 (2015) 158, [arXiv:1505.05488 [hep-ph]].

[2] ATLAS and CMS Collaborations, arXiv:1606.02266 [hep-ex].

[3] ATLAS Collaboration, Eur. Phys. J. C 76 (2016) 45, [arXiv:1507.05930 [hep-ex]]. CMS Collaboration, JHEP 1510144 (2015), [arXiv:1504.00936 [hep-ex]].

[4] ATLAS Collaboration, Phys. Rev. D 92032004 (2015), [arXiv:1509.04670 [hep-ex]]. CMS Collaboration, Phys. Lett. B 749560 (2015), [arXiv:1503.04114 [hep-ex]].

[5] A. Thamm, R. Torre and A. Wulzer, JHEP 1507100 (2015), [arXiv:1502.01701 [hep-ph]].

[6] ATLAS Collaboration, arXiv:1606.03833 [hep-ex]. CMS Collaboration, arXiv:1606.04093 [hep-ex].

[7] D. Buttazzo, A. Greljo and D. Marzocca, Eur. Phys. J. C 76 (2016) 116, [arXiv:1512.04929 [hep-ph]].

[8] R. Barbieri, D. Buttazzo, K. Kannike, F. Sala and A. Tesi, Phys. Rev. D 87, 115018 (2013), [arXiv:1304.3670 [hep-ph]].

[9] R. Barbieri, D. Buttazzo, L. J. Hall and D. Marzocca, arXiv:1603.00718 [hep-ph].

[10] D. Pappadopulo, A. Thamm, R. Torre and A. Wulzer, JHEP 1490 (2014) 060, [arXiv:1402.4431 [hep-ph]].

[11] M. Bellomo, talk at Rencontres de Moriond 2016, EW session.

[12] A. Greljo, G. Isidori and D. Marzocca, JHEP 1507 (2015) 142, [arXiv:1506.01705 [hep-ph]].

[13] D. Buttazzo, A. Greljo, G. Isidori and D. Marzocca, arXiv:1604.03940 [hep-ph].

[14] ATLAS Collaboration, JHEP 1507 (2015) 157, [arXiv:1502.07177 [hep-ex]].

[15] CMS Collaboration, Phys. Rev. D 91, 052009 (2015), [arXiv:1501.04198 [hep-ex]]. 